\title{
El uso de las TIC como herramienta de motivación para alumnos de enseñanza secundaria obligatoria. Estudio de caso español
}

\section{The use of ICTs as a motivational tool for students in compulsory secondary education. A spanish case study}

\author{
Antonio Jesús Amores-Valencia ${ }^{1}$ \\ https://orcid.org/0000-0001-9298-6548 \\ Patricia de-Casas-Moreno ${ }^{2}$ \\ http://orcid.org/0000-0003-1205-8106
}

Universidad de Nebrija, España

Recibido: 28-09-2019

Aceptado: 07-12-2019

\section{Cita recomendada}

Amores-Valencia, A. \& De-Casas-Moreno, P. (2019). El uso de las TIC como herramienta de motivación para alumnos de enseñanza secundaria obligatoria estudio de caso Español. Hamut'ay, 6(3), 37-49. http://dx.doi.org/10.21503/hamu.v6i3.1845

\section{RESUMEN}

Las Tecnologías de la Información y la Comunicación (TIC) se han alzado a día de hoy como un instrumento de gran influencia en la sociedad actual, sobre todo, en el contexto educativo. En este sentido, el presente estudio se centra en analizar la influencia de los recursos y herramientas digitales en la educación con la finalidad de motivar a los estudiantes en el aula. En relación a la metodología, se ha llevado a cabo una investigación exploratoria, optando por una metodología cuantitativa con el uso de las encuestas como herramienta de recolección de datos. Se ha contado con la participación de un total de 120 estudiantes de centros públicos, privados y concertados de la etapa de Educación Secundaria Obligatoria en la provincia de Málaga (España). Entre los resultados se puede destacar que los alumnos inciden en que su motivación aumenta gracias a la utilización de las TIC. No obstante, es necesario hacer uso de estas herramientas con el desarrollo de unas correctas competencias y habilidades digitales. Asimismo, se puede señalar, según los resultados, que la formación de los docentes no es la adecuada, ya que muchos de los discentes desconocen sobre las múltiples herramientas y aplicaciones para el desarrollo educativo. En suma, el análisis de este estudio va a ofrecer información detallada, útil y necesaria para enfocar el proceso de enseñanza y aprendizaje de una manera diferente.

Palabras Clave: Motivación, TIC, educación, competencia digital, herramientas digitales.

1 Graduado en Ingeniería Mecánica por la Universidad de Málaga. Ingeniero Técnico Industrial especializado en Mecánica. Experto Universitario en Ingeniería Mecánica. Máster Universitario en Tecnologías de la Información y la Comunicación para la Educación y el Aprendizaje Digital por la Universidad Antonio de Nebrija. Máster Universitario en Profesorado de Enseńanza Secundaria Obligatoria y Bachillerato, Formación Profesional y Enseńanza de Idiomas en la especialidad de Tecnología, Informática y Procesos Industriales. Docente en Educación Secundaria en el Colegio Cerrado de Calderón (Málaga). amoresvalencia@gmail.com

2 Doctora en Comunicación en la rama de Alfabetización Mediática por la Universidad de Huelva. Licenciada en Periodismo. Máster de Comunicación y Educación Audiovisual, especializada en Comunicación 2.0 y Redes Sociales. Miembro del Grupo Comunicar y del Consejo Técnico de la Revista Comunicar. Forma parte del Grupo de Investigación «Ágora» (HUM-648). Editora adjunta de la Revista Científica Universitaria «Ágora Digital». Su línea de investigación está centrada en calidad de los medios de comunicación, los estereotipos, el lenguaje informativo y los hábitos de la audiencia como punto de partida de propuestas educativas para un mejor consumo por parte de la sociedad. pcasas@nebrija.es 


\section{Abstract}

Information and Communication Technologies (ICTs) have become nowadays an instrument of great influence in today's society, especially in the educational context. In this sense, the present study focuses on analyzing the influence of digital resources and tools in the educational field in order to motivate students in the classroom. Regarding the methodology, an exploratory research has been carried out. We have selected a quantitative methodology with the use of surveys as a data collection tool. We had the participation of a total of 120 students from public, private and state-funded private institutions belonging to the compulsory Secondary Education in the province of Malaga (Spain). Among the results, it is possible to emphasize that the students recognize that their motivation increases thanks to the use of ICTs. However, it is necessary to make use of these tools along with the development of appropriate digital skills and abilities. It can also be noted, according to the results, that teacher training is not adequate, since many of them are unaware of the multiple tools and applications for the educational development. In short, the analysis of this study will offer detailed, useful and necessary information to approach the teaching and learning process in a different way.

Keywords: Motivation, ICTs, education, digital competence, digital tools.

\section{INTRODUCCIÓN}

A lo largo de la historia la sociedad se ha ido transformando debido a la introducción de la tecnología en ámbitos económicos, culturales o educativos. Por lo tanto, no se puede considerar la educación bajo un punto de vista estático, pero tampoco mostrarse ajena a las nuevas herramientas y dispositivos tecnológicos existentes. Debido a esto, el proceso de enseñanza y aprendizaje debe evolucionar y adaptarse de manera paralela a la sociedad para atender las diferentes demandas.

Las Tecnologías de la Información y la Comunicación (TIC) son recursos que han conllevado un gran efecto en la vida cotidiana, involucrando a todos los ámbitos de la sociedad. Esto supone la necesidad de prepararse para las nuevas particularidades que se derivan de su uso. En la sociedad de la información actual, donde el acceso a la misma se ha convertido en una constante, se han originado nuevos puestos de trabajo que requieren de una formación específica en el campo de las TIC. A causa de esto, la educación debe implicarse y evolucionar ante estas nuevas circunstancias, fomentando y proporcionando el acceso a estas herramientas y recursos digitales al alumnado. No obstante, con el fin de informar de manera adecuada y correcta estos conocimientos a los estudiantes para fomentar su motivación, es imprescindible que los docentes se formen de acuerdo con las necesidades y exigencias actuales. De acuerdo con lo expresado por Hernández (2017), es necesario trabajar la motivación con el alumnado, de modo que, no infiera un impedimento para la enseñanza de los conocimientos, así como evitar que se convierta en una dificultad para los docentes en su desempeño diario de impartir clases. Sin duda, hay que prestar atención a que los educandos pierden la atención. En este ambiente de desmotivación las TIC juegan un rol importante debido a su gran influencia como activadora de la motivación. Según lo expuesto por Macías y Manresa (2013), los niveles de motivación y el grado de interés que presentan los estudiantes se ven alterados ante el uso de las TIC. Así queda reflejado cómo estas herramientas tecnológicas tan usadas en su vida diaria consiguen favorecer el aprendizaje del alumnado. Además, resulta muy recomendable la práctica en el aula para adquirir las competencias digitales que ayuden a dar respuesta a las nuevas necesidades educativas. 
Sin duda alguna, hay que destacar el papel de los docentes a la hora de implantar estas nuevas tecnologías en el aula, ya que produce en estos unos profundos cambios a la hora de aplicar diferentes metodologías activas.

Las TIC se han incrementado a un ritmo sin precedentes en el campo educativo. Sin duda, hasta la actualidad, no se podría entender el proceso de enseñanza-aprendizaje sin ellas, debido al poder motivador e influencia positiva que transmite tanto a estudiantes como docentes. De este modo, el presente estudio tiene como objetivo general analizar el nivel de motivación de los estudiantes ante el desarrollo de contenido a través de las TIC en la etapa de Educación Secundaria obligatoria. Asimismo, como objetivos específicos se encuentran:

- Descubrir qué nivel de motivación que presentan los estudiantes cuando utilizan las TIC.

- Conocer qué herramientas y recursos digitales emplean en las aulas los estudiantes.

- Mostrar qué importancia tiene para los estudiantes las TIC en el proceso de enseñanza-aprendizaje.

- Detectar qué grado de formación tienen los estudiantes en el campo de las TIC.

Utilización de las tecnologías de la información y la comunicación y la motivación

En la actualidad, la utilización de las TIC está presente en la mayoría de las actividades que realizan las personas a diario. Esto implica que la sociedad debe estar preparada para adquirir nuevas competencias sociales y profesionales, y así, no quedarse al margen de este profundo proceso que se está produciendo. En particular en el ámbito educativo, se necesita una formación permanente del profesorado para otorgar a los estudiantes las competencias necesarias en el contexto mencionado. Los estándares educativos deben velar por la adquisición de conocimientos y habilidades que puedan ayudar a los discentes en la gestión del conocimiento. El docente debe ser el guía a través de estos nuevos medios, asegurando un desempeño profesional de alta calidad. La formación en competencias es una necesidad imperativa en el currículo educativo, sobre todo, si nos referimos a las competencias digitales para hacer uso de las
TIC. Esto, requiere una formación adecuada para trabajar paralelamente con el sistema educativo bajo las premisas de la sociedad red (INTEF, 2017). Además, las TIC están logrando la creación y transmisión de conocimientos que pueden ser compartidos a nivel mundial. De esta manera, se forma una sociedad abierta, en la que se puede mejorar ininterrumpidamente, gracias a la continua innovación en los procesos de enseñanza y aprendizaje. Este puede considerarse como uno de los principales motivos de la incursión de las TIC en las aulas, ya que ha pasado de ser recomendable a casi imprescindible.

La competencia digital juega un papel muy relevante en el proceso de enseñanza-aprendizaje. Sin embargo, no debe ser abordado como una materia en particular, sino integrada en todo el conjunto de materias. Según Area, Cepeda y Feliciano (2018), el proceso de aprendizaje tiene que partir de las TIC, ya que la mayoría de los estudiantes se encuentran familiarizados con ellas, obteniendo una mayor motivación. En este estudio realizado a más de 4.000 estudiantes de primaria y secundaria de todas las islas de Canarias, se abordaron cuestiones como el análisis de las características personales de los participantes, sus opiniones y valoraciones sobre las TIC, y sus efectos sobre el aprendizaje. El estudio comprendía todos los niveles educativos, y únicamente el $39 \%$ de los estudiantes coinciden con la etapa secundaria obligatoria. Para la recolección de datos se diseñó un cuestionario basado en 18 ítems, repartido y cumplimentado de manera online. Entre los resultados más destacados, sé evidenció que existe una relación entre la motivación y el uso de las TIC. Los alumnos de la etapa secundaria obligatoria obtienen un mayor interés sobre el aprendizaje a través de las TIC; 57.9\% siempre les gusta, $39.7 \%$ unos días les gusta y otros se aburren y $2.4 \%$ se aburren. Asimismo, hay una relación entre el género y la motivación por realizar trabajos con las nuevas tecnologías. Extrapolando los datos para la etapa estudiada, se obtiene que los varones prefieren realizar sus tareas con las TIC en mayor medida, concretamente en un $58.4 \%$, siendo un $41.6 \%$ para las mujeres. Por último, se aprecia una relación entre disponer o no de un ordenador para su uso en casa y la utilización de recursos tecnológicos en clase. En 
El uso de las TIC como herramienta de motivación para alumnos de enseñanza secundaria obligatoria. Estudio de caso español

este aspecto, sólo un $3.8 \%$ y un $10.8 \%$ respectivamente siempre se aburren realizando tareas en clase, mientras que un $62.9 \%$ y un $55 \%$ respectivamente afirman que les entusiasma trabajar en clase con dichos recursos.

Dadas todas estas relaciones, se puede destacar que los alumnos tienen más voluntad por aprender, en definitiva, mayor motivación e interés si su aprendizaje estaba basado en las TIC. Así, el uso de las tecnologías conlleva un gran impacto ,debido al cambio de metodología en las aulas, a los nuevos modelos pedagógicos aplicados, lo cual requiere una formación permanente de los docentes (Freeman et al., 2017).

Rol del docente como motivador en la utilización de las tecnologías

Los docentes desempeñan un papel muy relevante en la educación actual, ya que no se trata de enseñar los contenidos sino de abarcar el grado de motivación y de voluntad del alumnado. $\mathrm{Nu}$ merosas nociones sobre el rol que desempeñan los docentes en el uso de las TIC para la obtención de la competencia digital por parte de los alumnos, vienen reflejadas en el BOE, y concretamente en el Anexo II de la 738 Orden ECD/65/2015, de 21 de enero. En ellas, se refleja la potenciación de metodologías activas y contextualizadas, que consigan mayor motivación de los estudiantes. Además, los docentes deben mantener una actitud positiva hacia la búsqueda de innovación con estas herramientas para mejorar el modelo pedagógico y ayudar a los estudiantes en su aprendizaje.

La última legislación en España sobre las TIC, la Ley Orgánica 8/2013, de 9 de diciembre y el Real Decreto 1105/2014, de 26 de diciembre, señalan la importancia de las herramientas de aprendizaje en la Educación Secundaria Obligatoria y su integración en los centros educativos debe ser un hecho. Sin embargo, existen razones para pensar que queda un largo camino en la creación y uso de contenidos educativos virtuales como se constata en el informe español del programa para la evaluación internacional de alumnos (PISA, 2015). Cada tres años, este programa examina el grado de preparación en diferentes áreas temáticas de estudiantes con una edad comprendida entre los 15 y 16 años con el objetivo de evaluar la efectividad de los diferentes sistemas educativos e intentar captar los avances que se producen. Un resultado palpable de este informe es la falta de la competencia digital en los alumnos, el aumento de la motivación ante el uso de las tecnologías y la implicación del docente en todo el proceso.

En base a esto último, los docentes deben tener una gran variedad de estrategias para hacer frente a las diferentes situaciones que se presentan y a un aprendizaje cada vez más diverso y complejo. Se ha pasado de una sociedad industrial, donde se educaba a través de procesos mecánicos, a una sociedad del conocimiento en la que hay que desarrollar habilidades para resolver problemas. Asimismo, es importante tener una actitud crítica, gran capacidad de reflexión y decisión, así como conceder un mayor esfuerzo ante las dificultades motivacionales y emocionales como el aburrimiento, la falta de interés, las bajas expectativas y el alto grado de estrés de los estudiantes (Rodríguez et al., 2010). En este punto, el docente debe elegir el papel que juegan las tecnologías en el aula. Para ello, debe cumplir una serie de capacidades como, por ejemplo, la tendencia hacia el trabajo colaborativo y la presencia de las nuevas tecnologías en el contexto educativo. Asimismo, el profesor debe procurar empoderar a los alumnos con la finalidad que estos adquieran capacidades de reflexión y desarrollo crítico, que sepan cuestionar el funcionamiento del sistema, que trabajen bajo el principio de la horizontalidad o que intercambien mensajes entre sus pares. Por último, debe jugar un papel proactivo en el aula, apostando por las humanidades digitales. Estas son comprendidas como el conjunto de disciplinas humanísticas que, junto con el uso de las TIC, desarrollan nuevos paradigmas disruptivos con el objetivo de incluir críticamente el pensamiento humanista en la construcción digital y tecnológica de la sociedad (Rodrigo-Cano, de-Casas-Moreno \& Aguaded, 2018).

Según Castañeda, Esteve \& Adell, (2018), es necesario formar mejor a los docentes en relación a los conocimientos, habilidades y actitudes necesarias para promover el aprendizaje del alumno en este ecosistema identificado por las tecnologías. Sin duda, la formación docente todavía se plantea 
como un reto, habiendo surgido en estos últimos años el concepto de competencia digital docente.

Por otro lado, el proceso de aprendizaje no puede ser nunca considerado como una tarea única y exclusiva de los docentes, sino como un compendio entre profesores y estudiantes. Según ONTSI (2014a), los alumnos desempeñan una función primordial en el aprendizaje. En este punto es donde se encuentra la problemática actual, ya que esto implica una verdadera motivación para realizar las diferentes tareas, dada la baja participación e interés de los estudiantes que carecen de motivación ha causado un aumento del fracaso escolar.

Múltiples autores como Lazo (2012) indican que la organización de las sesiones y la realización de actividades, incluyendo los procedimientos y recursos facilitados por el docente, constituyen las estrategias motivacionales y promueven el aprendizaje significativo. Esto ayuda a conseguir los mejores resultados posibles y alcanzar objetivos tales como el acceso a la información, la adquisición de competencias y habilidades, la formación de actitudes y valores, siguiendo unas técnicas o pautas de trabajo. Por su parte, Arana, Meilán, Gordillo \& Carro (s.f.) describen las estrategias motivacionales como aquellas que deben potenciar la curiosidad del estudiante por los contenidos y tareas a realizar, otorgándoles un dominio sobre ellas. Además, deben ilustrar al alumnado sobre la importancia del contenido o la tarea en la adquisición de habilidades y competencias. Por último, orientar la atención de los alumnos hacia el proceso, buscando y desarrollando todos los pasos que se han dado, desde su idea hasta su valoración y conclusión.

En cuanto al actuar del docente en el aula, su función principal es ser mediador, y a la vez disponer de recursos, materiales concretos, metodologías (flipped classroom, ABP, design thinking, aprendizaje colaborativo, gamificación) y actividades, para lograr que el aprendizaje sea adquirido y conseguir oportunidades de comunicación entre los estudiantes. Para llevar a cabo esto, Campos (2010) propone la utilización de la retroalimentación (feedback) a través de ejercicios y actividades que incorporen el aprendizaje explícito (discusiones grupales, debates, lecturas,...) como el apren- dizaje implícito (proyectos, juegos, experiencias, ...), otorgando al docente los indicadores necesarios para la evaluación del aprendizaje. Bajo estas premisas, el docente debe velar por la coherencia entre sus objetivos y los del estudiante, activar el interés al comienzo de la clase, mantener la curiosidad promoviendo desafíos o retos motivadores, recurrir a ejemplos que se desarrollen en su contexto, impulsar la autonomía y lograr la adquisición de las metas propuestas.

La actitud hacia las TIC es un constructo que ha sido estudiado por diversos investigadores desde muy diferentes perspectivas, aunque sobre todo se ha trabajado bajo un componente cognitivo, afectivo y actitudinal (Torres, 2011). Sin embargo, el punto de partida es la propia motivación de los docentes, siendo conscientes de la relevancia del papel que juegan como agentes motivadores. Según Castela (2008), los docentes deben tener una personalidad propia capaz de crear nuevos escenarios en el aula, generando un clima apropiado y así aumentar el interés del alumnado para potenciar al máximo sus capacidades. Todas estas situaciones creadas, son de vital importancia a la hora de transmitir, ya que el alumnado es capaz de adquirir una mayor predisposición si observa una coherencia y credibilidad en el docente. Sin embargo, no sólo el profesor es el culpable de la motivación de los alumnos, sino que tiene que generarse en el interior de cada uno de ellos. A raíz de lo anteriormente mencionado, es necesario indicar una serie de pautas de actuación por parte de los docentes para afrontar las TIC con un espíritu motivador y una buena actitud: aprender de los errores que se comentan; realizar actividades educativas que fomenten la motivación por el trabajo; fomentar objetivos a corto plazo para lograr la satisfacción de los alumnos; comparar y compartir actividades y dudas con otros docentes para superar los problemas que surjan; desarrollar una formación actual en TIC; y planificar una programación innovadora y creativa (Castellano, 2012). 
El uso de las TIC como herramienta de motivación para alumnos de enseñanza secundaria obligatoria. Estudio de caso español

\section{Materiales y Métodos}

\section{Participantes}

El presente estudio estuvo conformado por una población de 120 alumnos. Los criterios de inclusión para colaborar en esta investigación consistían en pertenecer a la etapa educativa de Educación Secundaria Obligatoria de la provincia de Málaga (España). Se contó con la participación de cuatro centros educativos: Colegio Cerrado de Calderón (concertado), Colegio La Reina (concertado), Colegio San José de la Montaña (concertado) y IES Casabermeja (público). Cada uno de estos centros atienden al Real Decreto 1105/2014, de 26 de diciembre (BOE de 3 de enero de 2015), por el que se establece el currículo básico de la Educación Secundaria Obligatoria y del Bachillerato (art. 1-12), así como el artículo 3 del Decreto 111/2016, de 14 de junio (BOJA de 28 de junio), por el que se establece la ordenación y el currículo de la Educación Secundaria Obligatoria en la Comunidad Autónoma de Andalucía (art.13-14). La selección de la muestra se identifica por ser aleatoria sin atender a estratificaciones o perfiles determinados.

\section{Tipo y diseño de estudio}

La investigación se enmarca dentro del tipo cuantitativa, haciendo una recopilación de información, a través del uso de encuestas, con la finalidad de conseguir unos resultados reales y verídicos de lo que está aconteciendo actualmente en el aula de los centros educativos españoles. Según Del Canto y Silva (2013), este tipo de método emplea la recolección de datos para manifestar que los objetivos antepuestos se han considerado. Igualmente, certifican que es significativo partir de una base numérica y el análisis estadístico para instaurar modelos de comportamiento y probar teorías, confirmando la rigurosidad del proceso de investigación. En este, mismo orden de ideas, la metodología cuantitativa llevada a cabo va a ofrecer un enfoque exploratorio-descriptivo, gracias a la exposición de la media y desviación típica de las respuestas.

\section{Instrumento}

En la elección del instrumento de análisis, se ha considerado lo referido por López \& Fachelli (2015), que las encuestas son unas técnicas de investigación científica cuyo objetivo es conseguir medidas sobre los conceptos que proceden de un problema concreto. En las encuestas hay que señalar debidamente los rasgos y condiciones, y la recogida de datos. Además, con la ayuda de dicha herramienta desarrollada se observan dos supuestos, que validarán el estudio: en primer lugar, los ítems proporcionados permitirán responder al objetivo y, en segundo lugar, con esta acción se busca la involucración de los estudiantes (Monje, 2011).

La encuesta está conformada por un total de 58 ítems intercalando preguntas abiertas y cerradas, además de una escala Likert. Estos ítems a su vez, están divididos en tres dimensiones: a) perfil socio-demográfico, b) conocimiento y uso de las TIC y c) motivación, competencias y habilidades gracias a las TIC (Tabla 1 ).

Tabla 1

Instrumento de análisis -Encuesta

\begin{tabular}{ll}
\hline Dimensión & İtems \\
\hline $\begin{array}{l}\text { a) Perfil socio-de- } \\
\text { mográfico }\end{array}$ & $\begin{array}{l}\text { Edad; género; curso; centro; tipo de alum- } \\
\text { nado. }\end{array}$ \\
\hline b) Conocimiento y & ¿De qué dispositivos tecnológicos dispo- \\
uso de las TIC & ne?; ¿Dónde suele utilizar estos dispositi- \\
& $\begin{array}{l}\text { vos?; ¿Dispone de una cuenta de correo } \\
\text { electrónico?; ¿Cuántas cuentas de correo }\end{array}$ \\
& $\begin{array}{l}\text { electrónico tiene activas?; ¿Dispone de } \\
\text { una cuenta en alguna red social?; ¿Sabe }\end{array}$ \\
& $\begin{array}{l}\text { que el límite legal para usar una red so- } \\
\text { cial en España es de 14 años?; ¿Cuán- }\end{array}$ \\
& $\begin{array}{l}\text { tas redes sociales tienes activas?; ¿En } \\
\text { qué lugares suele conectarse a Internet?; } \\
\text { ¿Cuántas horas dedica a Internet?; ¿Qué }\end{array}$ \\
& $\begin{array}{l}\text { tipo de actividades desempeña mien- } \\
\text { tras está conectado?; ¿Qué programas/ }\end{array}$ \\
& aplicaciones suele utilizar?; ¿Cuál es la \\
& formación que tiene sobre las nuevas tec- \\
& nologías?; ¿Con qué frecuencia utiliza las \\
& TIC en el aula? \\
\hline c) Motivación, & En torno a las TIC...Los recursos digitales \\
competencias & en los centros educativos son adecuados; \\
y habilidades & La formación de los docentes en el área \\
gracias a las TIC & de las TIC es la adecuada; Las TIC permi- \\
(Señale con una & ten adecuar las actividades a las circuns- \\
escala del 1 al 4, & tancias personales de cada alumno; Las
\end{tabular}




\begin{tabular}{ll}
\hline Dimensión & İtems \\
\hline siendo 1 totalmen- & TIC fomentan la motivación entre los \\
te en desacuerdo, & alumnos; Un uso responsable es funda- \\
2 desacuerdo, 3 & mental cuando se trabaja con TIC; El uso \\
de acuerdo, y 4 to- & de las TIC en el aula es garantía de éxito \\
talmente de acuer- & en el proceso de enseñanza-aprendizaje; \\
do su valoración & Ayudan a afianzar los conocimientos en- \\
respecto a cada & señados en clase; Son una herramienta \\
temática) & adecuada para utilizarla en el aula; Supo- \\
& nen una mayor implicación de las familias; \\
& Su uso en el aula implica más beneficios \\
& que desventajas. ¿Para qué utiliza las TIC \\
& dentro del aula?.; ¿Qué herramientas TIC \\
& conoce?; ¿Utiliza alguna de estas redes \\
& en el aula?.
\end{tabular}

Fuente: Elaboración propia (2019).

La encuesta ha sido validada por un total de 5 expertos en materia de educación con ayuda de una rúbrica de evaluación cuya máxima puntuación es 40 puntos. De este modo, atendiendo a las cuestiones derivadas sobre estructura, contenido y formato de la herramienta, se ha conseguido obtener un total de 35,4 puntos. Este resultado demuestra la viabilidad de la encuesta para la recolección de datos. Asimismo, ha conseguido un promedio valorativo del $88.5 \%$ (Tabla 2 ).

Tabla 2

Rubrica de evaluación instrumento

\begin{tabular}{|c|c|c|c|c|c|c|}
\hline $\mathrm{N}^{\circ}$ & CRITERIOS & EV1 & EV2 & EV3 & EV4 & EV5 \\
\hline 1 & $\begin{array}{l}\text { Coherencia. ¿Las } \\
\text { preguntas elaboradas } \\
\text { tienen relación con } \\
\text { el título y con los } \\
\text { diferentes aspectos de } \\
\text { investigación? }\end{array}$ & 3 & 4 & 3 & 4 & 3 \\
\hline 2 & $\begin{array}{l}\text { Claridad. ¿La claridad } \\
\text { de las preguntas } \\
\text { es adecuada y se } \\
\text { entienden? }\end{array}$ & 3 & 4 & 3 & 4 & 3 \\
\hline 3 & $\begin{array}{l}\text { Metodología. ¿El } \\
\text { instrumento elaborado } \\
\text { responde al objetivo de } \\
\text { la investigación? }\end{array}$ & 4 & 4 & 3 & 4 & 3 \\
\hline 4 & $\begin{array}{l}\text { Suficiencia. ¿La } \\
\text { calidad y la cantidad } \\
\text { de preguntas son } \\
\text { adecuadas? }\end{array}$ & 3 & 4 & 2 & 3 & 3 \\
\hline 5 & $\begin{array}{l}\text { Experticia. ¿Existe una } \\
\text { relación del conoci- } \\
\text { miento de los encues- } \\
\text { tados con el contenido } \\
\text { del instrumento? }\end{array}$ & 4 & 4 & 3 & 3 & 4 \\
\hline
\end{tabular}

\begin{tabular}{|c|c|c|c|c|c|c|}
\hline $\mathrm{N} .^{\circ}$ & CRITERIOS & EV1 & EV2 & EV3 & EV4 & EV5 \\
\hline 6 & $\begin{array}{l}\text { Intencionalidad, } \\
\text { ¿Existe intencionalidad } \\
\text { expresada en las pre- } \\
\text { guntas expuestas? }\end{array}$ & 4 & 4 & 3 & 4 & 4 \\
\hline 7 & $\begin{array}{l}\text { Organización, ¿Existe } \\
\text { una secuencia lógica } \\
\text { y ordenada en las } \\
\text { preguntas? }\end{array}$ & 4 & 4 & 3 & 4 & 3 \\
\hline 8 & $\begin{array}{l}\text { Pertinencia, ¿Consi- } \\
\text { dera Ud. que las opcio- } \\
\text { nes empleadas son } \\
\text { correctas para medir } \\
\text { los diferentes aspectos } \\
\text { de la investigación? }\end{array}$ & 4 & 4 & 3 & 4 & 4 \\
\hline 9 & $\begin{array}{l}\text { Coherencia, ¿Hay } \\
\text { coherencia entre pre- } \\
\text { guntas en cuanto a la } \\
\text { forma y estructura? }\end{array}$ & 4 & 4 & 3 & 4 & 3 \\
\hline \multirow[t]{3}{*}{10} & $\begin{array}{l}\text { Actualidad, ¿Consi- } \\
\text { dera de actualidad el } \\
\text { tema tratado? }\end{array}$ & 3 & 4 & 4 & 4 & 3 \\
\hline & Porcentajes (\%) & 90 & 100 & 75 & 95 & 82.5 \\
\hline & Total/Media: 35.4 puntos & Tota & $(\%):$ & $.5 \%$ & & \\
\hline
\end{tabular}

Fuente: Elaboración propia (2019).

Por otro lado, una vez realizada la evaluación pertinente se ha llevado a cabo la fiabilidad del instrumento, obteniendo un alto grado de consistencia interna $(K=0.835)$. De este modo, según el alpha de cronbach se puede señalar que se trata de un instrumento fiable.

\section{Consentimiento informado}

La participación de los sujetos de la muestra ha sido de carácter anónimo, protegiendo sus datos personales. Los únicos datos que se han identificado son aquellos concernientes al perfil socio-demográfico (edad, género, curso escolar, etc). Cada uno de los estudiantes han participado libremente y han consentido el análisis de sus respuestas.

\section{Procedimiento}

El estudio se desarrolló en tres fases bien enmarcas:

I Fase: Contextualización teórica del estudio a través de la revisión bibliográficas en bases de datos de rigor académico. Esta primera fase, ayudó 
a identificar las necesidades y problemas del contexto educativo y estipuló las líneas y/o dimensiones de estudio para el instrumento de análisis.

II Fase: Desarrollo de la encuesta como herramienta de recolección de datos. Validación y evaluación por parte de los expertos. Recolección de datos, atendiendo a la muestra de estudio seleccionada para concluir con el análisis de resultados.

III Fase: Exposición e interpretación de los resultados obtenidos. Por último, se estableció la vinculación de los datos con los objetivos creados para este estudio, determinando las conclusiones más relevantes sobre el mismo.

\section{Resultados}

\section{Perfil socio-demográfico}

En lo que respecta a la edad de los estudiantes cuestionados, se puede destacar que hay una mayor prevalencia en la edad de 16 años (30.8\%), seguidos de aquellos de 14 años (23.3\%). Por su parte, los alumnos de $15(16.7 \%)$ y $13(16.7 \%)$ años han participado de manera equitativa. Sin embargo, son los alumnos de 12 años (12.5\%) los que han tenido un bajo grado de participación (Figura 1):

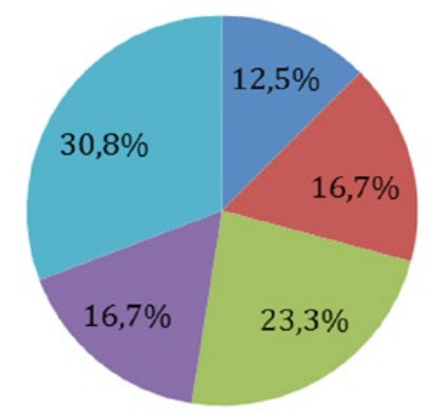

घ 12 años $\square 13$ años $\square 14$ años $\square 15$ años $\square 16$ años

Figura 1. Edad.

Fuente: Elaboración propia (2019).

Referente al género, predomina la categoría masculina $55.8 \%$ (67) frente a la femenina $44.2 \%$ (53). En relación a los cursos académicos, se evidencia como el curso de $4^{\circ}$ ESO es el grupo más numeroso con una representación del 34.2\% (41), seguido de $2^{\circ}$ ESO con un $24.2 \%$ (29). Son $3^{\circ} \mathrm{ESO}$ con un $21.7 \%$ (26) y $1^{\circ} \mathrm{ESO}$ con un $20 \%$ (24) aquellos que han obtenido una baja representación del alumnado. Asimismo, un 74.2\% (89) de los discentes cursan en centros concertados o privados, mientras que tan solo un $25.8 \%$ (31) de ellos pertenecen a centros públicos. Por último, dentro de esta dimensión, se ha querido valorar la posibilidad de si los estudiantes han repetido algún curso académico. Del total de alumnos encuestados, 115 no han repetido ningún curso (95.8\%), mientras que tan solo 5 estudiantes han repetido $(4.2 \%)$.

En resumen, el perfil representativo conseguido a través de este perfil socio-demográfico es el de un estudiante de 16 ańos, hombre, que cursa $4^{\circ}$ de la etapa educativa de Educación Secundaria Obligatoria en un centro concertado-privado y no ha repetido ningún curso académico.

\section{Conocimiento y uso de las TIC}

Profundizando en los resultados del estudio, se puede destacar que un $95.8 \%$ (115) de los encuestados declaran tener un Smartphone, siendo este el dispositivo más utilizado entre ellos. Además, los sujetos exponen que el portátil $87.5 \%$ (105) y la Tablet $78.3 \%$ (94) son los terminales más demandados después del Smartphone. A estos datos, hay que sumarle que un 99.2\% (119) de los alumnos han coincidido que hacen uso de estas herramientas con mayor frecuencia en el hogar en comparación con la escuela 22.5\% (27) (Figura 2).

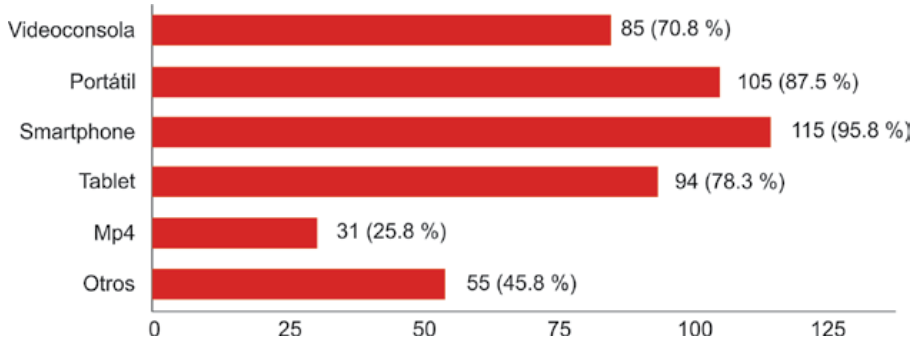

Figura 2. Dispositivos tecnológicos. Fuente: Elaboración propia (2019).

Por otro lado, atendiendo al uso y conocimiento de las nuevas tecnologías, se les cuestionó sobre 
la activación de las múltiples redes sociales existentes. Frente a ello, hay que tener en cuenta que cómo muchos de los estudiantes son menores de 14 años, era de obligado cumplimiento preguntar sobre su conocimiento de la edad mínima legal en Espańa para disponer de una cuenta. De este modo, se puede evidenciar como un 73.1\% (87) de los encuestados han manifestado que conocían dicho límite legal. En cambio, tan solo el 26.9\% (32) han seleccionado que no la conocían. Asimismo, en lo que se refiere al número de redes sociales activas, se puede subrayar que algo más de la mitad de la población encuestada, un $61.7 \%$ (74), tienen de 2 a 4 redes sociales activas, un $24.2 \%$ (29) tienen de 5 a 8 y tan solo el 9.2\% (11) expone que tiene más de 8 redes sociales activas en la actualidad. Por último, únicamente un $5 \%$ (6) afirman que tienen sólo una red social activa.

En la misma línea de estudio, hay que hacer hincapié a las horas que dedican a estar conectados a Internet y a las redes sociales, demostrando que un $40 \%$ (48) de los estudiantes se conectan diariamente entre 1 y 3 horas, seguidos de un $39.2 \%$ (47) que reconocen estar de tres a cinco horas diarias. Por último, un 20.8\% (25) de los encuestados indican que se conectan más de 6 horas a diario. Además, se puede apreciar cuáles son las acciones que desarrollan con mayor frecuencia, como, por ejemplo, hacer uso de las redes sociales con un $93.3 \%$ (112), seguido de los videojuegos 49.2\% (59) y la televisión 44. 2\% (53) (Figura 3).

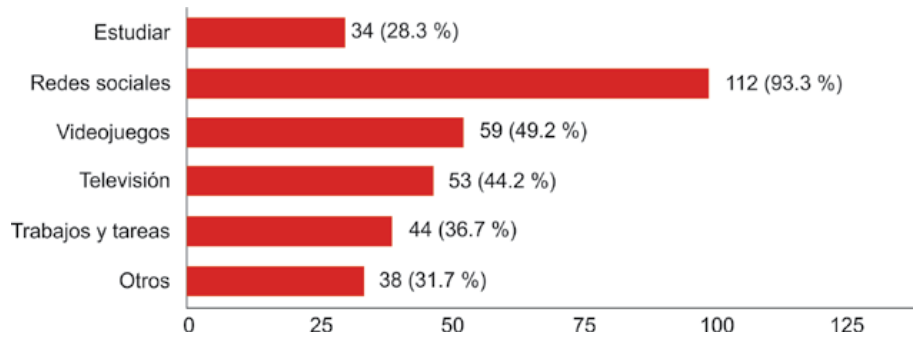

Figura 3. Utilización de las horas diarias que se dedica a Internet

Fuente: Elaboración propia (2019)

Por otro lado, se ha cuestionado sobre los programas y/o aplicaciones más populares o relevantes entre los estudiantes. De esta forma, se ha podido destacar que con un $100 \%$ (120), WhatsApp es la aplicación más popular, seguidamente de
Instagram y YouTube con un $92.5 \%$ (111). No obstante, aquellas aplicaciones con menor repercusión son Twitter con un 18.3\% (22), Skype con un $13.3 \%$ (16) y Facebook con un 5.8\% (Figura 4).

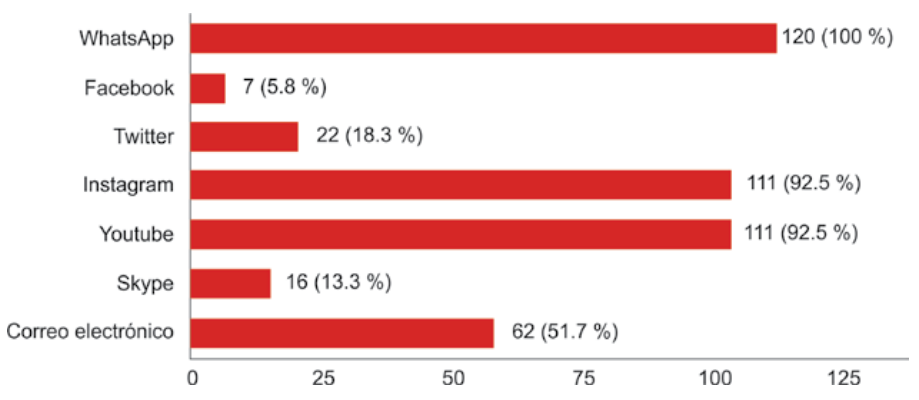

Figura 4: Programas y aplicaciones que se utilizan Fuente: Elaboración propia (2019)

Concerniente a la formación que poseen los encuestados en relación con las TIC, es necesario señalar que un $71 \%$ (86) afirman ser autodidactas, así como aprender a través de la práctica escolar con un 41.7\% (50). Además, un 52.5\% (63) de los alumnos han respondido utilizar las TIC en el aula casi nunca, mientras que un $24.2 \%$ (29) han mencionado que nunca suelen usar las herramientas tecnológicas. Tan solo un $12.5 \%$ (15) señalan hacer uso de las nuevas tecnologías a diario y únicamente el $10.8 \%$ (13) de las personas encuestadas han mencionado el uso habitual de las tecnologías en el aula.

Motivación, competencias y habilidades gracias a las TIC

Atendiendo al grado de motivación y conocimientos que tienen sobre las TIC, los valores de la media son cercanos a 3. Respecto a la desviación típica, se aprecia que los valores son cercanos a uno, por lo que no existen diferencias estadísticas significativas. En referencia a los valores más elevados, cabe destacar los ítems 23 (3.56), 22 (3.38), 26 (3.38) y 28 (3.31); los cuales están relacionados con las afirmaciones -Un uso responsable es fundamental cuando se trabaja con TIC-, -Las TIC fomentan la motivación entre los alumnos-, -Son una herramienta adecuada para utilizarla en el aula- y -Su uso en el aula implica más beneficios que desventajas-, respectivamente. Por su parte, los valores que han obtenido una 
El uso de las TIC como herramienta de motivación para alumnos de enseñanza secundaria obligatoria. Estudio de caso español

menor puntuación son los ítems 27 (2.43), 20 (2.61) y 21 (2.77), correspondientes a -Suponen una mayor implicación de las familias-, -La formación de los docentes en el área de las TIC es la adecuada- y -Las TIC permiten adecuar las actividades a las circunstancias personales de cada alumno-. En suma, se puede apuntar que los educandos valoran positivamente las TIC haciendo que su motivación incremente, aunque destacan negativamente la formación de los docentes en lo que se refiere a las nuevas tecnologías (Tabla 3).

Tabla 3:

En torno a las TIC

\begin{tabular}{cccccc}
\hline Ítems & $\mathrm{N}$ & Mínimo & Máximo & Media & Desv. Típica \\
\hline Ítem 19 & 120 & 1 & 4 & 2.86 & 0.853 \\
\hline Ítem 20 & 120 & 1 & 4 & 2.61 & 0.843 \\
\hline Ítem 21 & 120 & 1 & 4 & 2.77 & 0.914 \\
\hline Ítem 22 & 120 & 1 & 4 & 3.38 & 0.832 \\
\hline Ítem 23 & 120 & 1 & 4 & 3.56 & 0.683 \\
\hline Ítem 24 & 120 & 1 & 4 & 2.92 & 0.875 \\
\hline Ítem 25 & 120 & 1 & 4 & 3.24 & 0.830 \\
\hline Ítem 26 & 120 & 1 & 4 & 3.38 & 0.812 \\
\hline Ítem 27 & 120 & 1 & 4 & 2.43 & 1.059 \\
\hline Ítem 28 & 120 & 1 & 4 & 3.31 & 0.818 \\
\hline
\end{tabular}

Fuente: Elaboración propia (2019)

Por otro lado, planteando la utilidad de las TIC dentro del aula, se observa que la desviación típica refleja valores cercanos a uno por lo que no se aprecian diferencias estadísticas significativas. En relación con la media se muestran valores entre el 2 y el 3. Los ítems mejor valorados han sido el 31 (3.02), 34 (2.90) y 29 (2.65), correspondiendo a -Buscar información en Internet-, -Explicar contenidos- y -Ver vídeos-. En cambio, los ítems menos valorados han resultado ser el 33 (2.04), 30 (2.43) y 32 (2.45), aludiendo a -Realizar juegos-, -Ejercicios interactivos- y -Utilizar aplicaciones-. En síntesis, se podría decir que los estudiantes dentro de la etapa de Educación Secundaria Obligatoria no suelen utilizar herramientas digitales dentro del aula para fines que no sean explicar contenidos, buscar información o ver vídeos (Tabla 4).

Tabla 4:

Uso de las TIC en el aula

\begin{tabular}{cccccc}
\hline Ítems & $\mathrm{N}$ & Mínimo & Máximo & Media & Desv. Típica \\
\hline Ítem 29 & 120 & 1 & 4 & 2.65 & 1.105 \\
\hline Ítem 30 & 120 & 1 & 4 & 2.43 & 1.097 \\
\hline Ítem 31 & 120 & 1 & 4 & 3.02 & 1.021 \\
\hline Ítem 32 & 120 & 1 & 4 & 2.45 & 1.099 \\
\hline Ítem 33 & 120 & 1 & 4 & 2.04 & 1.118 \\
\hline Ítem 34 & 120 & 1 & 4 & 2.90 & 1.008 \\
\hline
\end{tabular}

Elaboración propia (2019)

En referencia al conocimiento que poseen los estudiantes sobre las múltiples herramientas TIC, la desviación típica presenta unos valores cercanos a uno por lo que no se aprecian diferencias estadísticas significativas. En cuanto a la media, cercanas a 3, se observan valores dispares, es decir, algunas de las aplicaciones son muy populares, en cambio, otras no son conocidas. Los ítems que presentan mejor evaluación son el 36 (3.88), 38 (3.49), 37 (3.40) y 35 (3.33), siendo estos -YouTube-, -Microsoft Office-, -Libre Office- y -Edmodo-. Sin embargo, los ítems menos populares son el 49 (1.18), 40 (1.22), 48 (1.36) y 39 (1.38), -Powtoon-, -Padlet-, -Symbaloo- y-Mentimeter-. Los resultados obtenidos, muestran un conocimiento bastante disímil entre las aplicaciones que se pueden utilizar dentro del aula (Tabla 5).

Tabla 5:

Conocimiento de herramientas TIC

\begin{tabular}{cccccc}
\hline Ítems & $\mathrm{N}$ & Mínimo & Máximo & Media & Desv. Típica \\
\hline Ítem 35 & 120 & 1 & 4 & 3.33 & 1.110 \\
\hline Ítem 36 & 120 & 1 & 4 & 3.88 & 0.459 \\
\hline Ítem 37 & 120 & 1 & 4 & 3.40 & 0.854 \\
\hline Ítem 38 & 120 & 1 & 4 & 3.49 & 0.789 \\
\hline Ítem 39 & 120 & 1 & 4 & 1.38 & 0.779 \\
\hline Ítem 40 & 120 & 1 & 4 & 1.22 & 0.553 \\
\hline Ítem 41 & 120 & 1 & 4 & 2.78 & 1.154 \\
\hline Ítem 42 & 120 & 1 & 4 & 2.97 & 1.209 \\
\hline Ítem 43 & 120 & 1 & 4 & 1.55 & 0.969 \\
\hline Ítem 44 & 120 & 1 & 4 & 3.18 & 0.961 \\
\hline Ítem 45 & 120 & 1 & 4 & 1.57 & 1.019 \\
\hline Ítem 46 & 120 & 1 & 4 & 1.89 & 1.019 \\
\hline Ítem 47 & 120 & 1 & 4 & 1.50 & 0.979 \\
\hline Ítem 48 & 120 & 1 & 4 & 1.36 & 0.765 \\
\hline Ítem 49 & 120 & 1 & 4 & 1.18 & 0.608 \\
\hline Ítem 50 & 120 & 1 & 4 & 1.58 & 0.993 \\
\hline
\end{tabular}

Fuente: Elaboración propia (2019) 
Por último y destacando cuáles son las redes sociales que se utilizan en el aula, la desviación típica presenta unos valores cercanos a uno por lo que no se aprecian diferencias estadísticas significativas. La media extraída, cercanas a 2, de las respuestas revela que el ítem más valorado es el 54 (2.63) -YouTube-. El resto obtiene unos valores por debajo de 2, destacando los ítems 58 (1.09), 51 (1.12) y 52 (1.12) -Vimeo-, -Facebook- y Twitter-. Tras haber analizado los resultados se aprecia que el uso de las redes sociales en el aula es un tema que está a años luz de su instauración, creando incluso dudas entre los estudiantes. Así, la única herramienta digital y social destacable se refiere al uso de vídeos (Tabla 6).

Tabla 6:

Las redes sociales en el aula

\begin{tabular}{cccccc}
\hline Ítems & $\mathrm{N}$ & Mínimo & Máximo & Media & Desv. Típica \\
\hline Ítem 51 & 120 & 1 & 4 & 1.12 & 0.522 \\
\hline Ítem 52 & 120 & 1 & 4 & 1.12 & 0.522 \\
\hline Ítem 53 & 120 & 1 & 4 & 1.68 & 1.159 \\
\hline Ítem 54 & 120 & 1 & 4 & 2.63 & 1.181 \\
\hline Ítem 55 & 120 & 1 & 4 & 1.56 & 1.052 \\
\hline Ítem 56 & 120 & 1 & 4 & 1.78 & 1.245 \\
\hline Ítem 57 & 120 & 1 & 4 & 1.40 & 0.902 \\
\hline Ítem 58 & 120 & 1 & 4 & 1.09 & 0.430 \\
\hline
\end{tabular}

Fuente: Elaboración propia (2019)

\section{Discusión y Conclusiones}

De acuerdo a los resultados y confrontándolos con las investigaciones estudiadas en el corpus de este estudio (Area, Cepeda, \& Feliciano, 2018; Freeman et al., 2017); se concluye que se ha podido verificar el objetivo general al ultimar que el dispositivo más popular es el Smartphone, siendo utilizado mayoritariamente en el hogar, entre 1 y 3 horas diarias para ver las redes sociales principalmente. Además, se ha constatado que todos los encuestados disponen de una red social y que conocen sobre el límite legal etario en España para su uso. Por otro lado, manifiestan que la aplicación por excelencia es el WhatsApp, aunque la formación autodidacta que han adquirido los estudiantes les ha permitido utilizar otras aplicaciones $y$ que la inmensa mayoría (76.7\%) casi nunca usan las TIC en el aula.

Otro aspecto relevante encontrado en los resultados es que los estudiantes aprecian positivamente el uso de las TIC dentro del aula, ya que esto desarrollaría su motivación e intervención en clase. Sin embargo, presentan una gran inexperiencia respecto al uso de muchas aplicaciones multimedia. Esto hace que surjan dudas a la hora de trabajar en el aula con muchas de las redes sociales, excluyendo YouTube. Resultados que coinciden con Colás, Reyes y Conde, (2018), al mencionar que el uso de las TIC en las aulas promueve la motivación estudiantil. No obstante, para ello es necesario que el docente tenga una formación adecuada sobre la implementación y uso de las tecnologías en los procesos de enseñanza-aprendizaje.

Hay que destacar que el uso de los dispositivos tecnológicos se centra, mayoritariamente, para realizar búsqueda de información, jugar a videojuegos o interaccionar a través de las redes sociales. Sin duda, gracias a los dispositivos móviles y al término de ubicuidad, se puede estar conectado, comunicado e informado en cualquier lugar y en cualquier momento. De este modo, es necesario recalcar que en el ámbito educativo también se deberían usar estas tecnologías para crear contenidos digitales, interactuar en entornos de aprendizaje o participar en comunidades virtuales (ONTSI, 2014a; 2014b).

Centrándonos en estas afirmaciones, se puede extraer la conclusión a raíz de esta investigación que los estudiantes aumentan su motivación si trabajan con las TIC, ya sea en la elaboración de actividades, en la comprensión de conceptos o en la búsqueda de información. Pero, hay cierto desconocimiento de muchas aplicaciones, dado que gran parte del profesorado no está debidamente formado en las nuevas tecnologías y, por lo tanto, no las introduce en el aula (Mayorga, Madrid \& Núñez, 2011), lo cual también se ha podido contrastar en este estudio.

En este estudio, también se evidencia que los estudiantes afirman que la motivación aumenta durante el desarrollo de las clases si se trabaja con dispositivos y herramientas digitales. Sin duda, hay que apostar por una enseñanza participativa en la que se involucre al alumnado, y se le otorgue 
El uso de las TIC como herramienta de motivación para alumnos de enseñanza secundaria obligatoria. Estudio de caso español

el papel de protagonista de su propio aprendizaje. Por ello, siempre hay que buscar recursos y estrategias que atraigan su atención y motivación, y en este sentido, las TIC responden a estos mandatos y requerimientos. No obstante, estos recursos tecnológicos han de ser utilizados de manera adecuada y correcta, para así resultar de gran ayuda a la hora de trabajar, adquirir y afianzar conceptos en clase. Con el fin de adaptarse a estas nuevas realidades, se considera imprescindible que dentro del aula se utilicen estos recursos tecnológicos y que la formación de los docentes sea la adecuada. En este apartado, los estudiantes consideran que los docentes deben ampliar o actualizar los conocimientos (Park, Kim, Shon \& Shim, 2013; Lee, Lee \& Hwang, 2015).

En los tiempos actuales, es innegable el aumento y uso de las redes sociales gracias a la irrupción de las TIC, lo que ha permitido alcanzar dimensiones espectaculares. Redes sociales como WhatsApp, con un total del $100 \%$ de uso entre los encuestados, Instagram y YouTube, con un $92.5 \%$ cada una o Twitter, con tan solo el $18.3 \%$, donde la población comparte con sus amigos y conocidos diferentes momentos de su vida, a través de fotos, vídeos o comentarios. A pesar de esta información tan poco halagüeña, las herramientas existentes son una fuente inagotable de recursos, que ofrecen multitud de posibilidades. Estos recursos son vistos como un elemento integrador dentro del aula, que van a fomentar la implicación de los estudiantes y facilitar el proceso de enseñanza, ya que atraen la atención de los estudiantes y les ayudan a trabajar de manera diferente posibilitando el afianzamiento de los contenidos. Aunque, los estudiantes encuestados están inmersos en la era digital, es curioso ver cómo no muestran un gran conocimiento de muchas de las herramientas educativas que disponen.

\section{Agradecimiento}

Este trabajo se enmarca en 'Alfamed' (Red Euroamericana de Investigadores), con el apoyo del Proyecto I+D “YOUTUBERS E INSTAGRAMMERS: LA COMPETENCIA MEDIATICA EN LOS PROSUMIDORES EMERGENTES" (RTI2018-093303-B-I00), financiado por la Agencia Estatal de Investigación del Ministerio de
Ciencia, Innovación y Universidades de España y el Fondo Europeo de Desarrollo Regional (FEDER).

\section{REFERENCIAS BIBLIOGRÁFICAS}

Arana, J. M., Meilán, J. J., Gordillo, F. \& Carro, J (s.f.). Estrategias motivacionales y de aprendizaje para fomentar el consumo responsable desde la Escuela. R.E.M.E, Revista Electrónica de Motivación y Emoción, XIII (35-36). Recuperado de http://bit.ly/2DXDhiV

Area, M., Cepeda, O. \& Feliciano, L. (2018). Perspectivas de los alumnos de Educación Primaria y Secundaria sobre el uso escolar de las TIC. Revista Educatio Siglo XXI. 36(2), 229-253. https://doi.org/10.6018/j/333071

Campos, A. L. (2010). Neuroeducación: uniendo las neurociencias y la educación en la búsqueda del desarrollo humano. La educ@ción, Revísta Digital, (143), 1-14.

Castañeda, L., Esteve, F., \& Adell, J. (2018). ¿Por qué es necesario repensar la competencia docente para el mundo digital? Revista de Educación a Distancia, 56, 1-20.

https://doi.org/10.6018/red/56/6

Castela, A. (2008). Influencias de los estilos docentes sobre la motivación y el rendimiento académico en la práctica educativa. Innovación y experiencias educativas, 13, 1-8.

Castellano, A. (2012). El profesor, principal agente de la motivación (Trabajo Final de Máster). Logroño: Universidad Internacional de la Rioja. Recuperado de http://bit. ly/2WsRsUk

Colás, P., Reyes, S. \& Conde, J. (2018). Los usos de las TIC en las aulas como factor predictivo del estado emocional de los estudiantes. Revista Curriculum, 31, 9-30. https://doi.org/10.25145/j.qurricul.2018.31.001

Del Canto, E., \& Silva, A. S. (2013). Metodología cuantitativa: abordaje desde la complementariedad en ciencias sociales. Revista de Ciencias Sociales, 141, 25-34.

https://doi.org/10.15517/rcs.v0i141.12479

Freeman, A., Adams, S., Cummins, M., David, A. \& Hall, C. (2017). Informe Horizon. Texas: The New Media Consortium.

Hernández, R.M. (2017). Impacto de las TIC en la educación: Retos y Perspectivas. Propósitos y Representaciones, 5(1), 325-347.

https://doi.org/10.20511/pyr2017.v5n1.149

INTEF (2017). Marco Común de Competencia Digital Docente. Recuperado de http://bit.ly/2Ja9KXw

Lazo, L. (2012). Estrategias para la enseñanza y el aprendizaje de la química general para estudiantes de primer año de universidad. Revista Electrónica Diálogos Educativos, 12 (23), 66-89.

Lee, Y., Lee, J. \& Hwang, Y. (2015). Relating motivation to information and communication technology acceptance: 
elf-determination theory perspective. Computers in $\mathrm{Hu}$ man Behaviour, 51, 418-428. https://doi.org/10.1016/j. chb.2015.05.021

Ley Orgánica 8/2013, de 9 de diciembre, para la mejora de la calidad educativa. Boletín Oficial del Estado. Madrid, 10 de diciembre de 2013, núm. 295, 97.858 a 97.921. Recuperado de http://bit.ly/2Yeaw9w

López, P. \& Fachelli, S. (2015). Metodología de la investigación social cuantitativa. Barcelona: Universitat Autónoma de Barcelona. Recuperado de http://bit.ly/2JYWW62

Macías, L. \& Manresa, C. (2013). Mayores y nuevas tecnologías: Motivaciones y dificultades. Ariadna (1), 6-11. https://doi.org/10.6035/Ariadna.2013.1.2

Mayorga, M.J., Madrid, D. \& Núńez, F. (2011). La competencia digital de los docentes. Formación y actualización en Web 2.0. Etic@net, 9(11), 213-232

Monje, C. A. (2011). Metodología de la investigación cuantitativa y cualitativa. Neiva (Colombia): Universidad Surcolombiana.

ONTSI (2014 a). Las TIC en el aula. Una aproximación a nuestra realidad educativa. Observatorio Nacional de las Telecomunicaciones y de la Sociedad de la Información. Recuperado de http://bit.ly/2H8Tbb5

ONTSI (2014 b). Familia y TIC. Observatorio Nacional de las Telecomunicaciones y de la Sociedad de la Información. Recuperado de http://bit.ly/2Jp8I9e

Park, N., Kim, Y. C., Shon, H. \& Shim, H. (2013). Factors influencing smartphone use and dependency in South Korea. Computers in Human Behavior, 29(4), 1763-1770. https://doi.org/10.1016/j.chb.2013.02.008

PISA (2015). Programa para la evaluación internacional de los alumnos. Informe Español. Recuperado de http://bit. ly/2Vkh4GE

Real Decreto 1105/2014, de 26 de diciembre, por el que se establece el currículo básico de la Educación Secundaria Obligatoria y del Bachillerato. Boletín Oficial del Estado. Madrid, 3 de enero de 2015, núm. 3, 169 a 546. Recuperado de http://bit.ly/306JEtw

Rodrigo-Cano, D., de-Casas-Moreno, P., \& Aguaded, I. (2018). El rol del docente universitario y su implicación ante las humanidades digitales. index.comunicación, 8(2), 13-31.

Rodríguez, S., Valles, A., González, R. \& Núñez, J. C. (2010). Motivar enseñando. La integración de estrategias motivadoras en el currículo escolar. Madrid: CCS.

Torres, G.M. (2011). Alfabetización Digital y Actitudes hacia las TIC. (Tesis doctoral publicada). Barcelona: Universidad Autónoma. Recuperado de http://bit.ly/2Y8oN7m 